\title{
CNES - Chalmers - IAP - ONERA - XLIM activities in the domain of high RF power breakdown phenomena
}

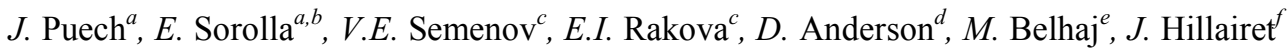 \\ ${ }^{a}$ CNES (France), 18 Avenue Edouard Belin, 31401 Toulouse, Cédex 9, France \\ ${ }^{\mathrm{b}}$ XLIM (France), 123 Avenue Albert Thomas, 87060 Limoges Cedex, France \\ ${ }^{\mathrm{c}}$ IAP (Russia), 46 Ulyanov St., Nizhny Novgorod, 603950, Russia \\ ${ }^{\mathrm{d}}$ Chalmers (Sweden), Chalmers University of Technology, SE-412 96 Göteborg, Sweden \\ ${ }^{e}$ ONERA (France), BP74025 - 2 Avenue Edouard Belin, 31055 toulouse, Cédex 4, France \\ ${ }^{\mathrm{f}}$ CEA, IRFM, F-13108 Saint Paul-lez-Durance, France
}

\begin{abstract}
Multipactor breakdown is an important potential failure mechanism in many different microwave devices working under close to vacuum conditions. Applications range from space borne RF equipment to high-power microwave generators. The basic physics involved in the multipactor phenomenon is well known for the case of two infinite pallel plates made of metal. However, most realistic RF device geometries involve inhomogeneous RF electric fields and curved field lines and sometimes also dielectric material. The purpose of this paper is to set up methodologies to determine the Multipactor threshold in such situations.
\end{abstract}

\section{Introduction}

The trend in the space telecommunication market is to increase the number of space segment users, these being spread over wide areas and asking for higher and higher data rate. At the payload level, more and more powerful microwave amplifiers are inserted in the chain. Components must handle very high RF electric fields in vacuum. Two kinds of discharges can occur depending on the pressure range:

- in a rarefied atmosphere, an avalanche-like increase of the electron density may occur due to secondary electron emission when electrons, accelerated by the RF electric field, hit the wall of the microwave device ( Multipactor effect)

- at finite pressures, a similar growth of the plasma density in the gas filling the device may occur due to impact ionization of neutral gas molecules by free electrons (Corona effect).

Risks of discharge such as Multipactor or Gaseous Discharge triggered by Multipactor can become critical and must be taken into account at payload level, as well as at equipment level. The consequences may be the link budget degradation or even damages to the equipment.

Different CNES projects involving the Institute of Applied Physics (Nizhny Novgorod - Russia), Chalmers
University of Technology (Göteborg - Sweden), XLIM/University of Limoges, ONERA and Centre National d'Etudes Spatiales (Toulouse - France) were working together on these phenomena. The purpose of this article is to describe methodologies which were setup to determine the Multipactor threshold in 2 cases: RF components with metal iris and RF components with dielectric material at the level of the critical gap.

\section{RF components with iris}

Iris geometry (fig. 1) is quite commonly used in microwave components.

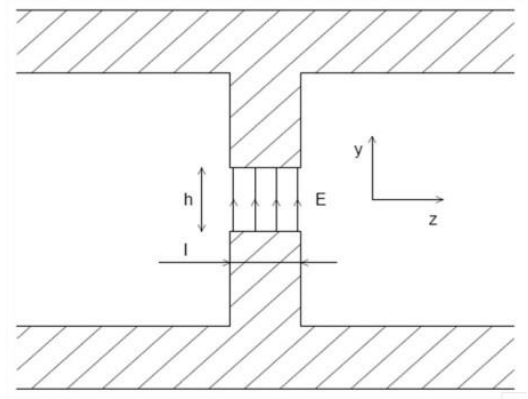

Fig. 1. Iris geometry.

In such situations, 3 physical phenomena have to be taken into account: 
- random walk of the electrons along the propagation axis,

- Miller force (ponderomotive force) near the iris edge,

- RF electric field component along the propagation axis

In this case, the spread in emission angles leads to a random walk of the corresponding electron trajectories that ultimately carries them out of the finite iris region and thus constitutes a loss mechanism that tends to increase the multipactor breakdown threshold. The importance of this diffusion out of the iris is determined by the ratio of the width, a, and height, $h$, of the iris and it was shown in [1] (assuming that the electric field was homogeneous inside the iris). The model is based on the statistical approach to determine the probability of surviving a certain number of gap crossings and thus the number of electrons after $\mathrm{k}$ steps. It enables to draw the the Multipactor chart as in the case of the 2 parallel plate model but with the ratio height over width ratio. The Figure 2 below illustrates this methodology for the alodine material (both sides of the iris) :

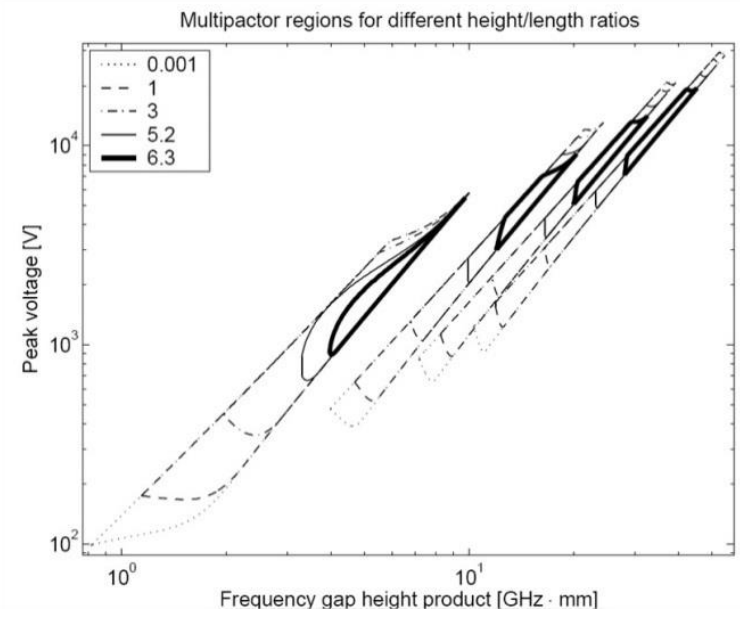

Fig. 2. Multipacor chart in the iris case.

A more rigorous and detailed analysis of the iris problem was made in [2] where the geometry of the iris was transformed into the classical 2 infinite parallel plate geometry using conformal mapping assuming that the electric field in the iris was approximately quasi-static. In parallel, a software called SEMA/MULSIM based on the Monte-Carlo approach was developed to determine the Multipactor threshold inside RF components with waveguide sections. Comparisons were drawn on the same RF component.

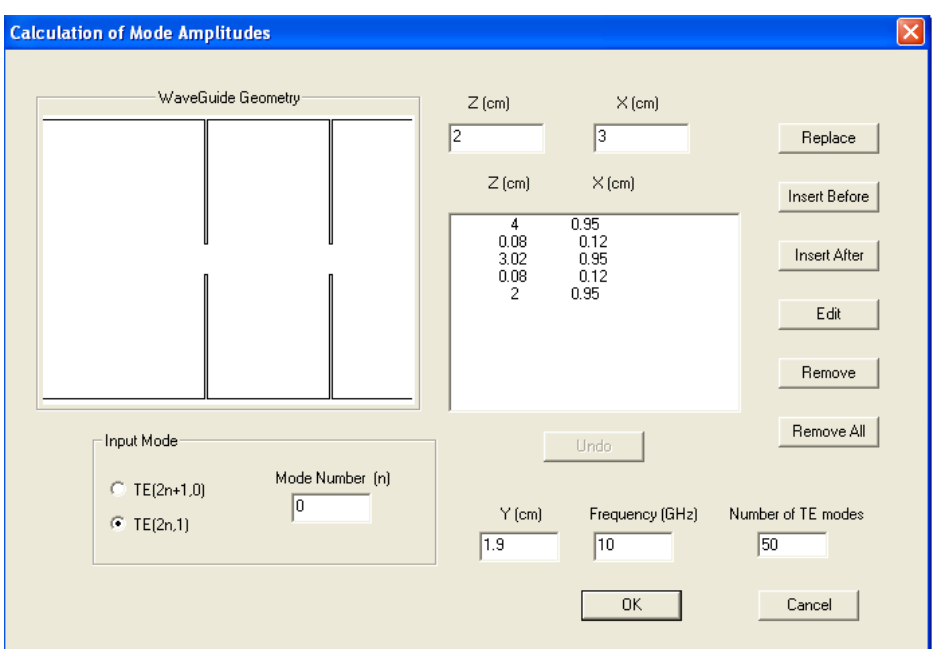

Fig. 3. RF component analysed with SEMA/MULSIM.

The figures 4 and figures 5 illustrates the number of electrons after $\mathrm{T}$ period versus the voltage $\mathrm{U}$. For the figure 4 , the calculations were realized with the SEMA/MULSIM software. For the figure 5, the calculations were realized with the conformal mapping approach.

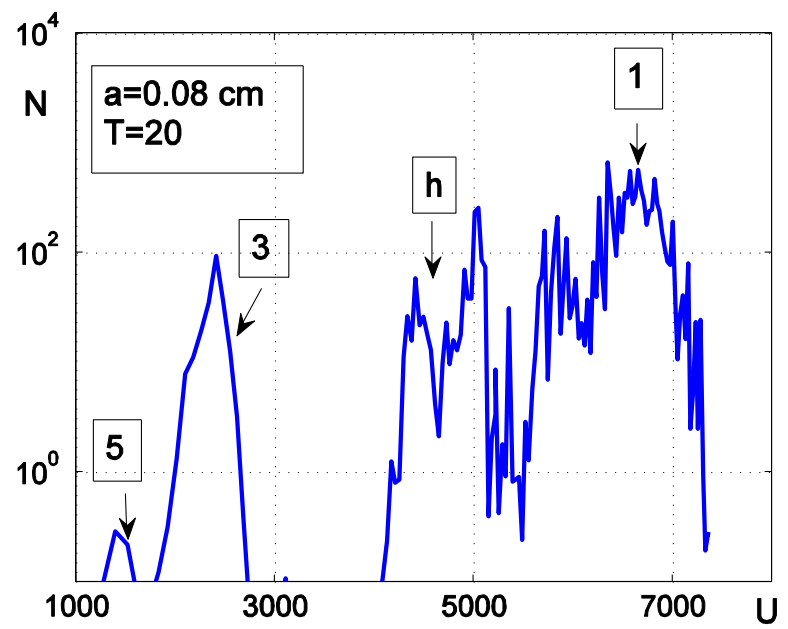

Fig. 4. Results obtained with the SEMA/MULSIM software.

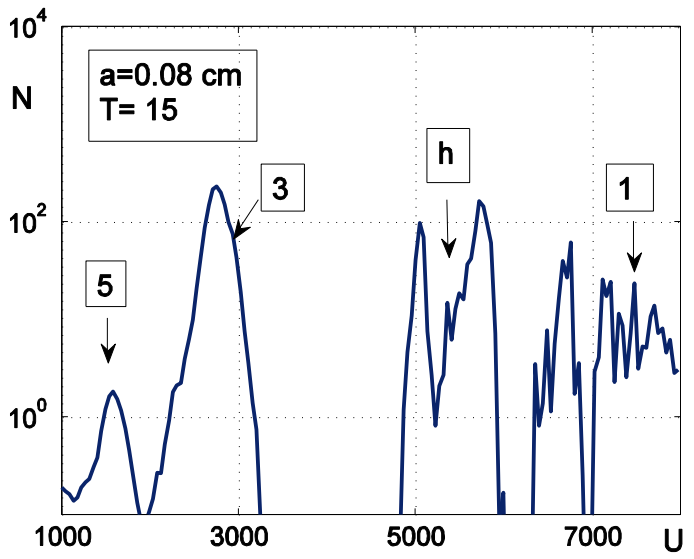

Fig. 5. Results obtained with the conformal approach. 
The 2 graphs are similar which demonstrates a good agreement between the 2 approaches.

Cross-validations were also performed between the conformal mapping approach and Multipactor measurements. These comparisons were performed within the EVEREST project between the conformal mapping software using ONERA secondary emission yield measurements of "technical" silver and Multipactor measurements of a $\mathrm{L}$ band coaxial filter with fringing field geometries [3]. The figure 6 shows the good agreement between the threshold found by measurement and the one found by the conformal mapping approach.

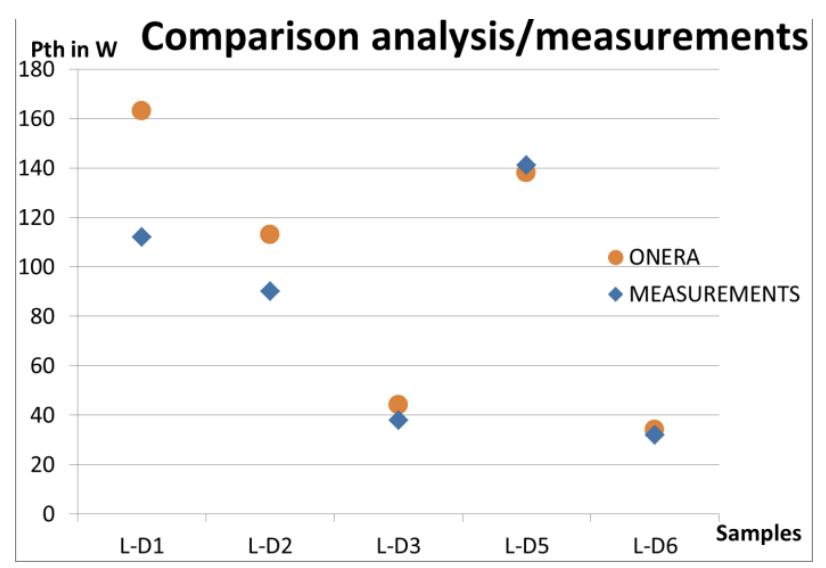

Fig. 6. Comparison between numerical simulations and measurements.

The agreement is excellent between the 2 approaches. It validates the conformal mapping methodology.

\section{RF components with dielectrics}

The trend in the space telecommunication payload is to reduce the footprint of the different high power RF components. One way to reach this goal is to use dielectric material inside these components, in order to lower the size while maintaining the overall RF performances. It is essential to determine the power capabilities of such components though Multipactor analysis. Thus it is necessary to study the electron dynamics in such situations.

Recent theories and secondary emission measurements at ONERA [4] have put in evidence the fact that the secondary emission properties of the dielectric varies with time leading to an equilibrium state. As a matter of fact, the secondary emission yield creates a positive or a negative charge which accelerates or decelerates the electrons before the collision. This charge reduces the SEY [5].

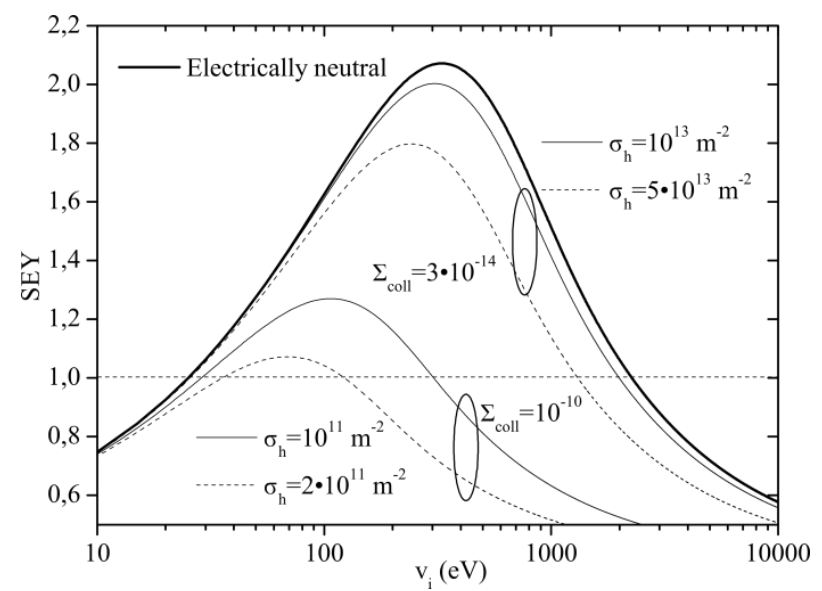

Fig. 7. Variation of the SEY with the surface density of holes.

Moreover, the charge created in the dielectric generates a DC field that modifies the force the electron cloud is subjected to. The electron dynamics within a 2 parallelplate geometry partially filled with dielectrics has been analyzed using a mono-energetic secondary emission model. The approximation of the electron cloud by a thin sheet of charge was done, what is valid slightly above the Multipactor voltage threshold. Both the decrease of the SEY with the charge in the dielectric and the subsequent electrostatic field generated are taken into account to find the equation of motion of the electron sheet when the waveguide is excited with the fundamental TM-mode.

When the dielectric filling factor of the waveguide is close to one, both the position and velocity of the electron cloud can be found analytically. The dimensionless equation for the position is as follows:

$$
\Lambda=\left[V_{\omega}-(\cos \alpha-\Omega \alpha)\right](\varphi-\alpha)+\sin \varphi-\sin \alpha-\frac{\Omega}{2}\left(\varphi^{2}-\alpha^{2}\right)
$$
where the dimensionless position, $\Lambda \equiv m_{e} \omega^{2}\left(x^{\prime}-x_{0}\right) /|e| E_{0}$, electric field, $\Omega \equiv E_{d} / E_{0}$, and velocity, $V_{\omega} \equiv m_{e} \omega v_{0} /|e| E_{0}$ and the parameter $\alpha$ (phase of the electric field when the electron begins the flight) have been defined. When the charge on the dielectric is close to zero, $\Omega=0$. A mathematical expression to evaluate the Multipactor electric field breakdown as function of the dielectric charge has been obtained using the classical resonance theory.

$E_{0}^{t h}=\frac{2 m_{e} \pi f|\gamma|}{|e| \cos \lambda} \sqrt{1+\frac{\mu \sin \lambda}{\gamma}+\left(\frac{\mu}{2 \gamma}\right)^{2}}$

where $\gamma \equiv\left[N \pi\left(v_{0}-N \pi|e| E d / 2 m e \omega\right)-\omega X\right] / S, \mu \equiv\left(v i-v_{0}\right.$ $+N \pi|e| E d / m e \omega)$ and $S=((N \pi) 2+4)^{1 / 2}$, being $N$ the order of resonance. It must be reminded that this expression is valid when $\left(|E d| \ll\left|E_{0}\right|\right)$. 
The comparison between this expression and the numerical simulations is illustrated in the figure 8 . It shows the electric field threshold obtained with the simulations for different values of the initial density of holes together with the predictions of the classical resonance theory (eq. 1).

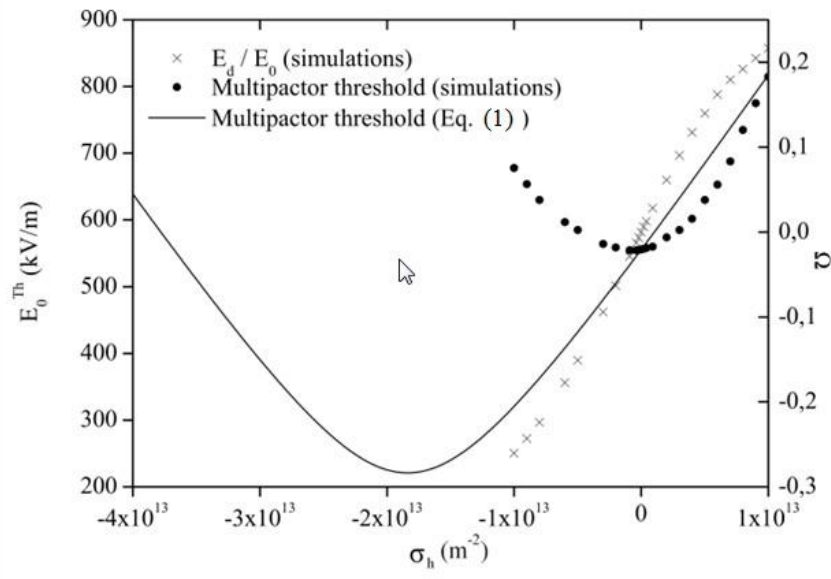

Fig. 8. Comparison of the electric field threshold obtained with the classical resonance theory and the simulations

When the dielectric charge is positive, the 2 methodologies do not differ too much. On the contrary when the dielectric charge is negative, the classicial theory underestimates the level of the Multipactor threshold. Some deeper studies need to be performed to understand such discrepancies. The mainpoint is that the simulations and the equation (1) predict a Multipactor power threshold that depends on the initial charge.

\section{Conclusion}

The problem of the iris geometry was treated in this paper. A first approach based on the random walk was depicted and enabled to draw Multipactor chart similar to the charts drawn in the parallel plate case but with the ratio height over width as parameter. The main drawback of this method is that it is too conservative as the 2 other electron losses mechanism as the Miller force and the fringing field effect are not taken into account. An approach proposed by V.E. Semenov and E.I. Rakova was set up. It was based on the conformal mapping methodology, taking into account the 3 electron losses mechanism. Comparisons of the Multipactor threshold on the same cases were drawn between the conformal mapping approach with the use of SEY measured data and measurements.A very good agreement could be observed what validates the approach.

This paper also focuses on the Multipactor threshold estimation inside RF samples with dielectric. It was observed that the conductivity and the subsequent charging phenomena of dielectrics have a strong impact on the Multipactor threshold determination. Based on theoretical elements, the dependence of the breakdown threshold on the ratio $\mathrm{E}_{\mathrm{DC}} / \mathrm{E}_{\mathrm{RF}}$ was given. The results presented enable to give an idea of the conductivity domain in which the "metal like" analysis is still valid.

As perspectives, the next step is to study RF components such as isolators/circulators and dielectric windows. This activity is performed in common with CEA IRFM and ONERA through a $\mathrm{PhD}$ student who focus on the influence of a DC magnetic field on the Multipactor. In that frame, a first inovant study [6] was realized and was dealing with the design of a SEY test set-up to quantify the influence of a DC magnetic field on the SEY characteristics of a given material sample.

\section{Acknowledgments}

The comparison between the conformal mapping simulations of iris and Multipactor measurements were realized in the frame of the TRP ESA funded activity EVEREST n²2452/09/NL/GLC

\section{References}

1. R. Udiljak, D. Anderson, M. Lisak, J. Puech, and V. E. Semenov, "Multipactor in a waveguide iris", IEEE Trans. Plasma Sci. Vol. 35, No. 2, pp. 388395, 2007.

2. V. E. Semenov, E. Rakova, R. Udiljak, D. Anderson, M. Lisak, J. Puech, "Conformal mapping analysis of multipactor breakdown in waveguide irises”, Phys. Plasmas, vol. 15, p. 033501, 2008.

3. J. Puech and EVEREST consortium, "Synthesis of the results of the EVEREST project", MULCOPIM 2014, Valencia, 17-19 Sept. 2014

4. M. Belhaj, D. Payan, J. Puech, "About the electron emission properties of dielectric materials", MULCOPIM 2017, Noordwijk, 5-7 April 2017.

5. E. Sorolla, M. Belhaj, J. Sombrin and J. Puech, Phys. Plasmas (unpublished).

6. N. Fil, M. Belhaj, J. Hillairet, J. Puech, R. Mathevet, "Electron Emission under Uniform Magnetic Field of Conductor Materials used for Spatial and Fusion Applications", MULCOPIM 2017, Noordwijk, 5-7 April 2017. 